\title{
SELEÇÃO RECORRENTE INTRAPOPULACIONAL NO MARACUJAZEIRO AMARELO:ALTERNATIVA DE CAPITALIZAÇÃO DE GANHOS GENÉTICOS
}

\author{
Intrapopulation recurrent selection in yellow passion fruit: alternative to accumulate genetic gains \\ Marcelo Geraldo de Morais Silva ${ }^{1}$, Alexandre Pio Viana ${ }^{2}$, Gustavo Menezes Gonçalves ${ }^{3}$, \\ Antonio Teixeira do Amaral Júnior ${ }^{4}$, Messias Gonzaga Pereira ${ }^{5}$
}

\begin{abstract}
RESUMO
Objetivou-se, neste trabalho, estimar parâmetros genéticos e correlações associadas a características agronômicas do maracujazeiro amarelo (Passiflora edulis Sims), predizer o progresso genético esperado com a seleção direta e baseado em índice de seleção, bem como sugerir uma estratégia de capitalização de ganhos genéticos nos ciclos de seleção recorrente. Foram avaliadas 26 progênies de meio-irmãos provenientes da recombinação de genótipos selecionados do primeiro ciclo de seleção via Delineamento I, as quais representam a população melhorada, UENF/MA. O delineamento estatístico utilizado foi blocos casualizados com duas repetições e parcelas constituídas de cinco plantas. Foram avaliadas cinco características relacionadas ao fruto, no período de fevereiro a julho de 2007, utilizando dados de média de parcelas. As estimativas dos parâmetros genéticos e das correlações, bem como a seleção baseada no índice de seleção possibilitaram a identificação de progênies com desempenhos agronômicos superiores para vários caracteres, simultaneamente. Além disso, a presente estratégia pode constituir alternativa de capitalização de ganhos genéticos na seleção recorrente, aumentando a eficiência do processo seletivo e potencializando a sua utilização em programas de melhoramento de fruteiras, uma vez que permite ganho genético em duas (geração e teste de progênies) das três etapas de seleção recorrente.
\end{abstract}

Termos para indexação: Passiflora edulis, índice de seleção, parâmetros genéticos.

\section{ABSTRACT}

The aim of this research was to estimate the genetic parameters and correlations associated to the agronomic traits of yellow passion fruit (Passiflora edulis Sims), to predict the expected genetic progress with the direct selection and based on selection index, as well as to suggest a strategy to accumulate genetic gains in the recurrent selection cycles. Twenty six half-sib progenies originated from the recombination of selected genotypes from the first cycle of selection via design I were evaluated, which represents the improved population, UENF/MA . The randomized complete block design with two replications and five plants per plot was used. Evaluations were made from February to July 2007 for five agronomic traits. Data of plot means were considered for statistical analysis. The genetic parameter estimates and correlations, as well as the selection based on the selection index made possible the identification of progenies with high performance for several traits, simultaneously. Besides, the strategy may constitute an alternative of accumulating genetic gains in yellow passion fruit recurrent selection, increasing the efficiency of the selective process and its use in fruit improvement programs, once it allows genetic gain in two (generation and progeny tests) of the three recurrent selection steps.

Index terms: Passiflora edulis, selection index, genetic parameters.

(Recebido em 22 de abril de 2008 e aprovado em 21 de agosto de 2008)

\section{INTRODUÇÃO}

O Brasil ocupa a posição de maior produtor e consumidor mundial de maracujá amarelo, sendo cultivado, no país, de acordo com estimativas do IBGE (2007) em torno de 480 mil toneladas, aproximadamente $70 \%$ do total produzido no mundo.
Apesar desse destaque, a produtividade nacional ainda é baixa, cerca de 13 t/ha, devido, principalmente, ao baixo emprego de tecnologia de produção e carência de populações geneticamente melhoradas (MELETTI \& MAIA, 1999).

Para corrigir os fatores limitantes da cultura, como a baixa produtividade, causada, dentre outros, pela grande

\footnotetext{
'Engenheiro Agrônomo, Doutorando em Produção Vegetal - Centro de Ciências e Tecnologias Agropecuárias/CCTA - Laboratório Melhoramento Genético Vegetal/LMGV - Universidade Estadual do Norte Fluminense Darcy Ribeio/UENF - Avenida Alberto Lamego, 2000 - Parque Califórnia - 28015-610 Campos dos Goytacazes, RJ - mcoro2003@yahoo.com.br - Bolsista Faperj

Engenheiro Agrônomo, Doutor em Produção Vegetal - Centro de Ciências e Tecnologias Agropecuárias/CCTA - Laboratório Melhoramento Genético Vegetal/LMGV - Universidade Estadual do Norte Fluminense Darcy Ribeio/UENF - Avenida Alberto Lamego, 2000 - Parque Califórnia - 28015-610 Campos dos Goytacazes, RJ - pirapora@uenf.br - Bolsista CNPq

3Engenheiro Agrônomo, Doutorando em Genética e Melhoramento de Plantas - Centro de Ciências e Tecnologias Agropecuárias/CCTA - Laboratório Melhoramento Genético Vegetal/LMGV - Universidade Estadual do Norte Fluminense Darcy Ribeio/UENF - Avenida Alberto Lamego, 2000 - Parque Califórnia - 28015-610 - Campos dos Goytacazes, RJ - mggustavo@yahoo.com.br - Bolsista Capes

${ }^{4}$ Engenheiro Agrônomo, Doutor em Genética e Melhoramento de Planta - Centro de Ciências e Tecnologias Agropecuárias/CCTA - Laboratório Melhoramento Genético Vegetal/LMGV - Universidade Estadual do Norte Fluminense Darcy Ribeio/UENF - Avenida Alberto Lamego, 2000 - Parque Califórnia - 28015-610 - Campos dos Goytacazes, RJ - amaraljr@uenf.br - Bolsista CNPq

${ }^{5}$ Engenheiro Agrônomo, Ph.D. em Melhoramento de Plantas - Centro de Ciências e Tecnologias Agropecuárias/CCTA - Laboratório Melhoramento Genético Vegetal/LMGV - Universidade Estadual do Norte Fluminense Darcy Ribeio/UENF - Avenida Alberto Lamego, 2000 - Parque Califórnia 28015-610 - Campos dos Goytacazes, RJ - messias@uenf.br - Bolsista CNPq
} 
variabilidade existente nos pomares e pela falta de genótipos adaptados a todas as regiões, o melhoramento genético se torna indispensável. Alguns trabalhos até então desenvolvidos têm dado atenção em alternativas de seleção (GONÇALVES et al., 2007), parâmetros genéticos (MORAES et al., 2005; VIANA et al., 2003a, 2004), estudos com as espécies relacionadas e suas características reprodutivas (MELETTI et al., 2003; SOUZA et al., 2004; VIANA et al., 2003b), além de estudos sobre diversidade genética em populações (GANGA et al., 2004; VIANA et al., 2006).

A seleção recorrente, como método de melhoramento intrapopulacional associado à ferramentas biométricas pode auxiliar na seleção de materiais genéticos superiores (HALLAUER \& MIRANDA FILHO, 1988). Contudo, informações dessa natureza são escassas e estudos com ênfase em biometria são necessários para que o melhoramento do maracujazeiro alcance maior sucesso.

No desenvolvimento de um programa de seleção recorrente, a seleção de progênies superiores é feita na fase de avaliação (fase de teste), em experimentos com repetição. Porém, o maracujazeiro amarelo permite seleção também na fase de geração das progênies (população melhorada), possibilitando-se maximizar os ganhos genéticos durante os ciclos da seleção recorrente.

Neste contexto, objetivou-se, nesse trabalho, apresentar uma estratégia para seleção recorrente em maracujazeiro amarelo (Passiflora edulis Sims), estimar parâmetros genéticos e correlações associadas a características agronômicas e predizer o progresso genético esperado com a seleção, evidenciando a possibilidade de ganhos genéticos na fase de geração das progênies.

\section{MATERIAL E MÉTODOS}

O experimento foi instalado em outubro de 2006, na área experimental da Escola Agrícola Antônio Sarlo, localizada no município de Campos dos Goytacazes, região Norte do estado do Rio de Janeiro, com latitude sul de $21^{\circ}$ $45^{\prime}$, longitude $41^{\circ} 20^{\prime} \mathrm{W}$ e $11 \mathrm{~m}$ de altitude.

Foram avaliadas 26 progênies de meio-irmãos provenientes da recombinação das progênies selecionadas por Gonçalves (2005), na condução do delineamento I de Comstock \& Robinson (1948). Essa recombinação foi feita utilizando meio-irmãos maternos, onde a mãe foi a própria progênie selecionada e o pai foi a mistura de pólen de todas as progênies selecionadas. Dessa forma, as progênies avaliadas representam a população de trabalho para a condução do segundo ciclo de seleção. A Figura 1 contém o esquema de condução do programa de seleção recorrente do maracujazeiro amarelo.

O delineamento estatístico utilizado foi blocos ao acaso, com duas repetições e parcela experimental constituída de cinco plantas. O sistema de condução utilizado foi o de espaldeira vertical, com mourões de 2,5 metros de altura, espaçados a 4 metros e com um fio de arame número 12 a 1,80 metros do solo. A distância entre linhas de plantio foi de 3,5 metros. Os tratos culturais foram os recomendados para a cultura do maracujazeiro.

As avaliações das progênies foram realizadas de fevereiro a julho de 2007, mensurando as seguintes características: número médio de frutos por planta (NF), avaliado pela contagem visual do número de frutos por parcela, provenientes da primeira florada, dividido pelo número de plantas da parcela; peso médio de frutos em gramas (PF), obtido por meio de pesagem em balança digital, amostrando quinze frutos por parcela; comprimento médio de frutos em milímetros (CF), obtido por meio de medidas das dimensões longitudinais dos frutos com paquímetro digital, amostrando quinze frutos por parcela; largura média de frutos em milímetros (LF), obtida por meio de medidas das dimensões transversais dos frutos com paquímetro digital, amostrando quinze frutos por parcela; espessura média de casca em milímetros (EC), obtida por meio de medidas de quatro pontos da casca externa, na porção mediana dos frutos com paquímetro digital, amostrando quinze frutos por parcela. Foram utilizadas as médias aritméticas das medidas (média por parcela) de frutos fisiologicamente maduros.

Para verificar a existência de variabilidade genética entre as progênies estudadas, foi realizada análise de variância e estimados os parâmetros genéticos da população. Para tais procedimentos foi utilizado o programa computacional Genes (CRUZ, 2006). O modelo estatístico adotado foi $\mathrm{Y}_{\mathrm{ij}}=\mathrm{m}+\mathrm{g}_{\mathrm{i}}+\mathrm{b}_{\mathrm{j}}+\varepsilon_{\mathrm{ij}}$, onde: $\mathrm{Y}_{\mathrm{ij}}=$ observação referente à progênie $i$ no bloco $\mathrm{j}, \mathrm{m}=$ média geral, $\mathrm{g}_{\mathrm{i}}=$ efeito da progênie $\mathrm{i}, \mathrm{b}_{\mathrm{j}}=$ efeito do bloco $\mathrm{j}, \mathrm{e}_{\mathrm{ij}}=$ erro associado à observação $\left(\varepsilon_{\mathrm{ij}} \sim \operatorname{NID}\left(0, \sigma^{2}\right)\right.$.

Para o cálculo dos coeficientes de correlação entre os pares de características foram utilizadas as expressões conforme Cruz \& Regazzi (2001), sendo $\mathrm{r}_{\mathrm{F}}=\mathrm{COV}_{\mathrm{F}(\mathrm{X}, \mathrm{Y})}$ / $\sigma_{\mathrm{FX}} \sigma_{\mathrm{FY}} \mathrm{er}_{\mathrm{G}}=\mathrm{COV}_{\mathrm{G}(\mathrm{X}, \mathrm{Y})} / \sigma_{\mathrm{GX}} \sigma_{\mathrm{GY}}$, onde: $\operatorname{COV}_{\mathrm{F}(\mathrm{X}, \mathrm{Y})}$ e $\operatorname{COV}_{\mathrm{G}(\mathrm{X}, \mathrm{Y})}$ correspondem, respectivamente, às covariâncias fenotípicas e genotípicas entre as características x e y; $\sigma_{\mathrm{FX}}$ e $\sigma_{\mathrm{GX}}$ correspondem ao desvio padrão fenotípico e genotípico da característica $\mathrm{x} ; \sigma_{\mathrm{FY}}$ e $\sigma_{\mathrm{GY}}$ correspondem ao desvio padrão fenotípico e genotípico da característica y.

Para a estimação dos ganhos genéticos preditos, foi empregado além da seleção direta nas características avaliadas, o índice de seleção de Mulamba \& Mock (1978). Esse índice consiste em classificar as progênies em relação a cada uma das características, em ordem favorável ao melhoramento, assim, as ordens referentes às características são somadas para cada progênie obtendo a soma de ranks. Dessa forma, quanto menor for o valor obtido, melhor é o desempenho de uma progênie em relação às várias características estudadas (CRUZ \& REGAZZI, 2001). 
$\mathrm{C}_{0}$

Aplicação do Delineamento I

2002 (DI) na população base (UENF$\left.\mathrm{MA}_{0}\right)$ - obtenção de 113 progênies de Irmãos Completos

\begin{tabular}{l|l|}
2004 & $\begin{array}{l}\text { Avaliação das progênies de IC } \\
\text { para características agronômicas } \\
\text { em ensaios em 3 locais e com } \\
\text { repetição. }\end{array}$ \\
2004 & $\begin{array}{l}\text { Seleção das 30\% melhores } \\
\text { progênies com base em índices } \\
\text { de seleção. }\end{array}$ \\
2006 & $\begin{array}{l}\text { Recombinação das progênies } \\
\text { selecionadas usando progênies } \\
\text { de MI - finalização do 10 } 1^{\circ} \text { ciclo } \\
\text { de seleção via DI. }\end{array}$ \\
\hline
\end{tabular}

2007

2007

2008

2008

2009
$\mathrm{C}_{1}$

$\mathrm{C}_{2}$
Nova população de trabalho $\left(\mathrm{UENF}_{\mathrm{MA}}\right)_{1}$ ) estruturada em delineamento estatístico (DBC).

\begin{tabular}{|c|}
\hline I \\
\hline $\begin{array}{l}\text { Avaliação de } 26 \text { progênies de } \\
\text { Meio-irmãos } \\
\text { com base nas características } \\
\text { agronômicas. }\end{array}$ \\
\hline
\end{tabular}

Obtenção de progênies de Irmãos Completos com base no desempenho de seus genitores (progênies de MI).

Avaliação das progênies de IC e testemunhas em experimento com repetição.

\section{I}

Seleção das $30 \%$ melhores progênies com auxílio de índices de seleção.

Recombinação das melhores progênies - Finalização do $2^{\underline{\underline{ }}}$ ciclo de seleção recorrente
Nova população de trabalho (UENF-MA $A_{2}$ ) estruturada em delineamento estatístico (DBC).

Figura 1 -Fluxograma do programa de seleção recorrente intrapopulacional do maracujazeiro amarelo. 
Foram simulados dois tipos de ganhos genéticos envolvendo as 26 progênies. O primeiro, selecionando as oito progênies superiores e o segundo, selecionando as 18 superiores.

\section{RESULTADOS E DISCUSSÃO}

Verificam-se, pelos quadrados médios, a existência de variabilidade genética significativa pelo teste $F$ para as características $\mathrm{PF}$ e LF, a $1 \%$ de probabilidade e para $\mathrm{CF}$ a $5 \%$ de probabilidade, indicando a possibilidade de seleção das progênies superiores (Tabela 1). Já para as características NF e EC as diferenças foram não significativas. Esse comportamento similar das progênies para NF pode ser explicado pelo crescimento inicial desuniforme das plantas, atribuída a uma doença que atacou as plantas na fase de mudas. Além disso, apenas uma mensuração de NF foi feita durante todo o período de avaliação, não traduzindo o que, de fato, foi observado no experimento. Para EC, a ausência de significância pode ser devido à falta de variabilidade genética entre as progênies, para essa característica.

A precisão experimental, avaliada por meio do coeficiente de variação (CV), apresentou valores de 2,22 $\%$ a $71,91 \%$ e estão em acordo com os valores obtidos por Gonçalves et al. (2007), Moraes et al. (2005) e Viana et al. (2004). O maior CV foi detectado pra NF e seu valor elevado pode ser resultado da desuniformidade ambiental da área experimental, das diferenças de vigor entre as progênies e também da presença de doenças na fase inicial.

As estimativas dos coeficientes de herdabilidade apresentaram valores de 36,9\% a 83,5\%. Nas características NF e EC os efeitos de progênies foram não significativos, sendo observados os menores valores de herdabilidade.
Para as demais características, os valores encontrados estão em conformidade com os observados em outros trabalhos (GONÇALVES et al., 2007; MORAES et al., 2005; VIANA et al., 2004) e indicam a possibilidade de seleção de progênies geneticamente superiores.

O conhecimento da correlação entre dois caracteres é de fundamental importância no melhoramento de qualquer cultura, pois facilita o processo de seleção em caracteres de difícil mensuração e identificação ou que apresentem baixa herdabilidade (CRUZ \& REGAZZI, 2001).

$\mathrm{Na}$ Tabela 2 estão expostas as estimativas dos coeficientes de correlações genéticas e fenotípicas simples entre os pares de características estudados. A característica $\mathrm{NF}$, não se correlacionou com nenhuma característica, indicando a possibilidade de selecionar progênies superiores em todos os caracteres avaliados. Viana et al. (2003b) encontraram resultados similares para o ambiente de Macaé e Campos dos Goytacazes e uma correlação positiva entre NF e PF para o ambiente de Campos. Moraes et al. (2005) não observaram associações entre NF e as características PF, CF e LF, entretanto, a correlação genética entre NF e produção de frutos foi alta e positiva, possibilitando a sua utilização na seleção indireta da produção de frutos.

As características NF e produção parcial podem ser utilizadas como indicadores do potencial produtivo das progênies, já que a produção total é de difícil mensuração, pois o período de colheita é longo e várias avaliações são necessárias para se obter estimativas mais precisas. Moraes et al. (2005) observaram que a produção total, medida em sete meses se correlacionou altamente com a produção medida nos três primeiros meses $(r=0,95)$. Por outro lado, Maluf et al. (1989) obtiveram baixa

Tabela 1 - Resumo da análise de variância individual para características agronômicas do maracujazeiro amarelo e alguns parâmetros genéticos importantes associados.

\begin{tabular}{lcccccc}
\hline \multicolumn{1}{c}{ Fonte de } & Graus de & \multicolumn{5}{c}{ Quadrados médios $^{(1)}$} \\
\cline { 3 - 7 } Variação & liberdade & NF & PF & CF & LF & EC \\
\hline Blocos & 1 & 466,176 & 54,223 & 34,020 & 1,877 & 0,328 \\
Progênies & 25 & $187,202^{\text {ns }}$ & $551,704^{* *}$ & $25,885^{*}$ & $16,387^{* *}$ & $2,655^{\mathrm{ns}}$ \\
Resíduo & 25 & 118,165 & 185,867 & 10,685 & 2,702 & 1,575 \\
\hline Média & - & 15,11 & 160,36 & 78,42 & 73,85 & 7,94 \\
CV $(\%)$ & - & 71,91 & 8,50 & 4,16 & 2,22 & 15,78 \\
DMS $(5 \%)$ & - & 34,61 & 56,05 & 13,43 & 6,75 & 5,16 \\
$\mathrm{~h}^{2}(\%)$ & - & 36,9 & 66,30 & 58,70 & 83,50 & 40,7 \\
\hline
\end{tabular}

(1) NF - número de frutos por planta; $\mathrm{PF}$ - peso de frutos, em g; CF - comprimento de frutos, em mm; LF - largura de frutos, em $\mathrm{mm}$; EC - espessura de casca, em mm. **, * ${ }^{\text {ns }}$ Significativo a $1 \%$ e $5 \%$ de probabilidade e não significativo, respectivamente, pelo teste $\mathrm{F}$. 
correlação entre produção total e produção das 10 primeiras semanas de colheita. Investigações posteriores são necessárias para verificação dessas associações, principalmente estudos que mensurem a produção ao longo de todo o ciclo produtivo da cultura.

Conforme Tabela 2, para as demais características (PF, CF, LF e EC), foram encontradas correlações positivas entre todos os pares, indicando que progênies com maior peso de frutos tendem a ter frutos maiores (longitudinalmente e transversalmente) e com maior espessura de casca.

Na Tabela 3, observam-se as estimativas dos ganhos genéticos preditos por meio da seleção truncada e do uso de índice de seleção, considerando duas simulações de ganho. Uma, selecionando oito indivíduos superiores e outra, selecionando 18 indivíduos superiores.

Pode-se observar que os ganhos diretos para cada característica individual foram maiores do que os obtidos pelo índice de seleção. Os ganhos genéticos preditos são diretamente proporcionais ao diferencial de seleção, ou seja, quanto menor o diferencial de seleção, menor o ganho genético, pois, aumenta-se o número de selecionados, contribuindo com a diminuição do valor da média. Dessa forma, tanto os ganhos diretos, quanto os fundamentados no índice são maiores quando se considera a seleção dos oito indivíduos superiores.

Ao selecionar progênies superiores para uma característica, mudanças indesejáveis podem ocorrer em outras, em razão da existência de correlações entre elas
(CRUZ \& REGAZZI, 2001). Os resultados mostram que a seleção com base em uma única característica é inadequada, pois conduz a um produto final superior com relação à essa característica, mas leva a desempenhos não tão favoráveis para as demais não consideradas. Cruz (2006) comenta que, na obtenção de materiais genéticos superiores, o melhorista necessita selecionar com base em vários caracteres, para melhor inferir sobre a superioridade relativa dos mesmos. Uma forma de se aumentar o êxito com a seleção é por meio da utilização dos índices de seleção, pois esses permitem a seleção simultânea de várias características de interesse. Gonçalves et al. (2007) obtiveram o melhor ganho genotípico predito utilizando o índice de Mulamba \& Mock (1978), em uma população de maracujá amarelo, estruturada no Delineamento I.

Na utilização do índice de seleção foi empregado peso econômico baseado no coeficiente de variação genético $\left(\mathrm{CV}_{\mathrm{g}}\right)$. Cruz (1990) recomenda a utilização de estatísticas dos próprios dados experimentais. Para o autor o $\mathrm{CV}_{\mathrm{g}}$ é referencial, pois é diretamente proporcional à variância genética, mantendo de certa forma, uma proporção entre os caracteres.

Verifica-se que, para a seleção das oito progênies superiores, os ganhos genéticos fundamentados no índice foram próximos da seleção direta, com exceção de comprimento do fruto, onde os ganhos foram menores e da espessura de casca, onde os ganhos foram levemente positivos para o aumento da espessura de casca (Tabela 3).

Tabela 2 -Estimativas dos coeficientes de correlação fenotípica $\left(\mathrm{r}_{\mathrm{F}}\right)$ e genética $\left(\mathrm{r}_{\mathrm{G}}\right)$ entre características do maracujazeiro amarelo.

\begin{tabular}{|c|c|c|c|c|c|}
\hline \multirow{2}{*}{\multicolumn{2}{|c|}{ Características ${ }^{(1)}$}} & \multicolumn{4}{|c|}{ Correlações } \\
\hline & & $\mathrm{PF}$ & $\mathrm{CF}$ & LF & $\mathrm{EC}$ \\
\hline \multirow[t]{2}{*}{$\mathrm{NF}$} & $r_{F}$ & $-0,0575$ & $-0,1796$ & 0,0064 & $-0,3725$ \\
\hline & $\mathrm{r}_{\mathrm{G}}$ & $-0,0384$ & $-0,7343$ & $-0,1112$ & $-1,6339$ \\
\hline \multirow[t]{2}{*}{$\mathrm{PF}$} & $\mathrm{r}_{\mathrm{F}}$ & - & $0,6485^{* *}$ & $0,8156^{* *}$ & $0,4931 *$ \\
\hline & $\mathrm{r}_{\mathrm{G}}$ & - & 0,6258 & 0,9144 & 0,8676 \\
\hline \multirow[t]{2}{*}{$\mathrm{CF}$} & $\mathrm{r}_{\mathrm{F}}$ & - & - & $0,5935^{* *}$ & $0,5266^{* *}$ \\
\hline & $\mathrm{r}_{\mathrm{G}}$ & - & - & 0,6013 & 0,7138 \\
\hline \multirow[t]{2}{*}{ LF } & $r_{F}$ & - & - & - & $0,4352 *$ \\
\hline & $\mathrm{r}_{\mathrm{G}}$ & - & - & - & 0,6575 \\
\hline
\end{tabular}

(1) NF - número de frutos por planta; $\mathrm{PF}$ - peso de frutos, em gramas; CF - comprimento de frutos, em mm; LF - largura de frutos, em mm; EC - espessura de casca, em mm. ** Significativo a $1 \%$ e $5 \%$ de probabilidade, respectivamente, pelo teste $\mathrm{t}$. 
Tabela 3 - Estimativas de ganhos genéticos preditos para 26 progênies de meio-irmãos em maracujazeiro amarelo, por meio de seleção direta e índice de seleção.

\begin{tabular}{ccccc}
\multirow{2}{*}{ Características $^{(3)}$} & \multicolumn{2}{c}{ Seleção direta } & \multicolumn{2}{c}{ Índice de seleção } \\
\cline { 2 - 5 } NF & GS $^{1}$ & $\mathrm{GS}^{2}$ & $\mathrm{GS}^{2}$ \\
\cline { 2 - 5 } $\mathrm{PF}$ & 2,97 & 1,19 & 2,47 & 1,03 \\
$\mathrm{CF}$ & 7,83 & 3,49 & 3,1 & 3,18 \\
LF & 2,79 & 1,27 & 0,64 & 0,47 \\
EC & 3,56 & 1,56 & 2,64 & 1,36 \\
& $-5,85$ & $-3,07$ & 0,64 & 0,65 \\
\hline
\end{tabular}

${ }^{(3)} \mathrm{NF}$ - número de frutos por planta; PF - peso de frutos, em gramas; CF - comprimento de frutos, em mm; LF - largura de frutos, em mm; EC - espessura de casca, em mm. GS ${ }^{1}$ e GS ${ }^{2}$ - ganho de seleção em porcentagem, considerando a seleção de 8 e 18 progênies superiores, respectivamente.

Quando se considerou a seleção dos 18 materiais de melhor performance, o objetivo foi a continuidade do programa de seleção recorrente. Para isso, utilizou-se uma pressão de seleção menor, para não comprometer a variabilidade dos ciclos subseqüentes da seleção recorrente. Por conseguinte, os ganhos genéticos preditos, mesmo que menores, foram expressivos e contribuíram com o processo de seleção. Além disso, as 18 progênies selecionadas foram empregadas como genitoras das progênies para a fase de teste na seleção recorrente num esquema de dialelo, onde foram obtidas 153 progênies. Dessa maneira, a informação desse trabalho poderá auxiliar programas de melhoramento de fruteiras no sentido de aumentar a eficiência do processo seletivo, maximizando os ganhos em duas das três etapas da seleção recorrente.

\section{CONCLUSÕES}

A estratégia de seleção na fase de geração de progênies permitiu a obtenção de ganhos genéticos.

As estimativas dos parâmetros genéticos e correlações, bem como os ganhos genéticos observados possibilitaram a seleção de progênies superiores de maracujazeiro.

\section{AGRADECIMENTOS}

À CAPES, pela concessão da bolsa de doutorado. À FAPERJ, pelo suporte financeiro para desenvolvimento do projeto.

\section{REFERÊNCIAS BIBLIOGRÁFICAS}

COMSTOCK, R. E.; ROBINSON, H. F. The components of genetic variance in populations of biparental progenies and their use in estimating the average degree of dominance. Biometrics, Washington, v. 4, p. 254-266, 1948.
CRUZ, C. D. Aplicação de algumas técnicas multivariadas no melhoramento de plantas. 1990. $188 \mathrm{f}$. Tese (Doutorado em Genética e Melhoramento) - Escola Superior de Agricultura Luiz de Queiroz, Piracicaba, 1990.

CRUZ, C. D. Programa genes: biometria. Viçosa: UFV, 2006. v. $1,382 \mathrm{p}$.

CRUZ, C. D.; REGAZZI, A. J. Modelos biométricos aplicados ao melhoramento genético. 2. ed. Viçosa: UFV, $2001.390 \mathrm{p}$.

GANGA, R. M. D.; RUGGIERO, C.; LEMOS, E. G. de M.; GRILI, G. V. G.; GONÇALVES, M. M.; CHAGAS, E. A.; WICKERT, E. Diversidade genética em maracujazeiroamarelo utilizando marcadores moleculares fAFLP. Revista Brasileira de Fruticultura, Jaboticabal, v. 26, n. 3, p. 494498, dez. 2004.

GONÇALVES, G. M. Estimativas de parâmetros genéticos em características produtivas de maracujazeiro amarelo (Passiflora edulis $f$. flavicarpa), baseado no delineamento I. 2005. 87 f. Dissertação (Mestrado em Produção Vegetal) - Universidade Estadual do Norte Fluminense Darcy Ribeiro, Campos dos Goytacazes, 2005.

GONÇALVES, G. M.; VIANA, A. P.; BARROS NETO, F. V.; PEREIRA, M. G.; PEREIRA, T. N. S. Seleção e herdabilidade na predição de ganhos genéticos em maracujá-amarelo. Pesquisa Agropecuária Brasileira, Brasília, v. 42, n. 2, p. 235-240, fev. 2007.

HALLAUER, A. R.; MIRANDA FILHO, J. B. Quantitative genetics in maize breeding. Ames: Iowa State University, 1988. $468 \mathrm{p}$. 
INSTITUTO BRASILEIRO DE GEOGRAFIA E ESTATÍSTICA. Lavouras permanentes. Rio de Janeiro, RJ, 2007. Disponível em: ' http://Www.sidra.ibge.gov.bry. Acesso em: 3 maio 2007.

MALUF, W. R.; SILVA, J. R.; GRATTAPAGLIA, D.; TOMA-BRAGHINI, M.; CORTE, R. D.; MACHADO, M. A.; CALDAS, L. S. Genetic gains via clonal selection in passion fruit Passiflora edulis Sims. Revista Brasileira de Genética, São Paulo, v. 12, n. 4, p. 833-841, 1989.

MELETTI, L. M. M.; BERNACCI, L. C.; SOARES-SCOTT, M. D.; AZEVEDO FILHO, J. A. Variabilidade genética em caracteres morfológicos, agronômicos e citogenéticos de populações de maracujazeiro-doce. Revista Brasileira de Fruticultura, Jaboticabal, v. 25, p. 275-278, 2003.

MELETTI, L. M. M.; MAIA, M. L. Maracujá: produção e comercialização. Campinas: Instituto Agronômico, 1999. 62 p. (Boletim técnico, 181).

MORAES, M. C. de; GERALDI, I. O.; MATTA, F. de P.; VIEIRA, M. L. C. Genetic and phenotypic parameter estimates for yield and fruit quality traits from a single wide cross in yellow passion fruit. HortScience, Dordrecht, v. 40, n. 7, p. 1978-1981, Dec. 2005

MULAMBA, N. N.; MOCK, J. J. Improvement of yield potential of the Eto Blanco maize (Zea mays L.) population by breeding for plant traits. Egyptian Journal Genetics and Cytology, Alexandria, v. 7, p. 40-51, 1978.

SOUSA, M. M. de; PEREIRA, T. N. S.; VIANA, A. P.; PEREIRA, M. G.; AMARAL JÚNIOR, A. T. do;
MADUREIRA, H. C. Flower receptivity and fruit characteristics associate to time of pollination in yellow passion fruit Passiflora edulis sims f. falvicarpa. Scientia Horticulture, v. 1, p. 1-13, 2004.

VIANA, A. P.; PEREIRA, T. N. S.; PEREIRA, M. G.; AMARAL JÚNIOR, A. T. do; SOUZA, M. M. de; MALDONADO, J. F. M. Parâmetros genéticos em populações de maracujazeiro amarelo. Revista Ceres, Viçosa, v. 51, n. 297, p. 541-551, 2004.

VIANA, A. P.; PEREIRA, T. N. S.; PEREIRA, M. G.; SOUZA, M. M. de; MALDONADO, J. F. M.; AMARAL JÚNIOR, A. T. do. Diversidade genética entre genótipos comerciais de maracujazeiro amarelo (Passiflora edulis f. flavicarpa) e entre espécies nativas de Passiflora determinada por marcadores RAPD. Revista Brasileira de Fruticultura, Jaboticabal, v. 25, p. 489-493, 2003 a.

VIANA, A. P.; PEREIRA, T. N. S.; PEREIRA, M. G.; SOUZA, M. M. de; MALDONADO, J. F. M.; AMARAL JÚNIOR, A. T. do. Simple and canonic correlation between agronomical and fruit quality traits in yellow passion fruit (Passiflora edulis f. flavicarpa) populations. Crop Breeding and Applied Biotechnology, Viçosa, v. 3, n. 2, p. 133-140, 2003b.

VIANA, A. P.; PEREIRA, T. N. S.; PEREIRA, M. G.; SOUZA, M. M. de; MALDONADO, J. F. M.; AMARAL JÚNIOR, A. T. do. Genetic diversity in yellow passion fruit populations. Crop Breeding and Applied ABiotechnology, Viçosa, v. 6, p. 87-94, 2006 